International Journal of Pure and Applied Mathematics

Volume 106 No. 3 2016, 965-976

ISSN: 1311-8080 (printed version); ISSN: 1314-3395 (on-line version)

url: http://www.ijpam.eu

doi: 10.12732/ijpam.v106i3.20

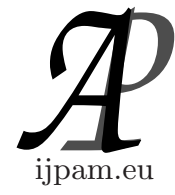

\title{
NEW ITERATIVE METHODS FOR SINGLE VARIABLE EQUATIONS
}

\author{
Chahn Yong Jung ${ }^{1}$, Arif Rafiq ${ }^{2}$, Faisal Ali $^{3}$, Shin Min Kang ${ }^{4}$ § \\ ${ }^{1}$ Department of Business Administration \\ Gyeongsang National University \\ Jinju, 52828, KOREA \\ ${ }^{2}$ Department of Mathematics and Statistics \\ Virtual University of Pakistan \\ Lahore, 54000, PAKISTAN \\ ${ }^{3}$ Center for Advanced Studies in Pure and Applied Mathematics \\ Bahauddin Zakariya University \\ Multan, 60800, PAKISTAN \\ ${ }^{4}$ Department of Mathematics and RINS \\ Gyeongsang National University \\ Jinju, 52828, KOREA
}

\begin{abstract}
In this paper, we establish new iterative methods for the solution of scalar equations by using the decomposition technique due to Noor and Noor [Some iterative schemes for nonlinear equations, Appl. Math. Comput. 183 (2006), 774-779].
\end{abstract}

AMS Subject Classification: $65 \mathrm{H} 05$

Key Words: iterative methods, nonlinear equations, convergence order, multiple roots

\section{Introduction}

One of the most significant problems in numerical analysis is to find the solution of nonlinear equations. Various iterative methods have been studied to find the approximate roots of nonlinear equations, see $[1,3,4,5,7,10,11,13]$ and references there in. These methods can be classified as one-step, two-step and

Received: December 8, 2015

Published: March 4, 2016

$\S$ Correspondence author (c) 2016 Academic Publications, Ltd. url: www.acadpubl.eu 
three-step methods. In [5], Chun has proposed several one-step and two-step iterative methods with higher-order convergence by using the decomposition technique of Adomian [2]. Many other iterative methods have also been developed for finding the simple roots of nonlinear equations. But there are many scientific problems in which the nature of the solution to the governing nonlinear equations is not simple. In order to find the multiple roots, Schröder [18] made the first contribution and introduced the following modified Newton's method:

$$
x_{n+1}=x_{n}-m \frac{f\left(x_{n}\right)}{f^{\prime}\left(x_{n}\right)} .
$$

If $m=1$, then the equation is the classical Newton's formula.

During the last two decades, much attention has been devoted by various researchers for solving nonlinear equation with multiple roots. Chun et al. [6], Chun and Neta [8], Homeier [12], Osada [17] have developed some techniques to find the multiple roots of nonlinear equations. In the recent years, the researchers have made significant and interesting contribution in this field $[9$, $14,15]$.

In this paper, we propose some new iterative methods for finding multiple roots of scalar equations by using decomposition technique given in [16].

Consider the nonlinear equation

$$
f(x)=0 .
$$

It is well known that if $\alpha$ is a root with multiplicity $m$, then it is also a root of $f^{\prime}(x)=0$ with multiplicity $m-1$ of $f^{\prime \prime}(x)=0$ with multiplicity $m-2$ and so on. Hence if initial guess $x_{0}$ is sufficiently close to $\alpha$, the expressions

$$
\begin{aligned}
& x_{0}-m \frac{f\left(x_{0}\right)}{f^{\prime}\left(x_{0}\right)}, \\
& x_{0}-(m-1) \frac{f^{\prime}\left(x_{0}\right)}{f^{\prime \prime}\left(x_{0}\right)}, \\
& x_{0}-(m-2) \frac{f^{\prime \prime}\left(x_{0}\right)}{f^{\prime \prime \prime}\left(x_{0}\right)},
\end{aligned}
$$

will have the same value.

Remark 1.1. The generalized Newton's formula

$$
x_{n+1}=x_{n}-2 \frac{f\left(x_{n}\right)}{f^{\prime}\left(x_{n}\right)},
$$


gives a quadratic convergence when the equation $f(x)=0$ has a pair of double roots in the neighbourhood of $x_{0}$. It may be noted that for the double root $\alpha$ near to $x_{0}, f(\alpha)=0=f^{\prime}(\alpha)$.

\section{New Iterative Methods}

We can rewrite the nonlinear equation (1.1) as a coupled system:

$$
\begin{gathered}
f(\gamma)+(x-\gamma) \frac{f^{\prime}(\gamma)+f^{\prime}(x)}{2}+g(x)=0, \\
g(x)=f(x)-f(\gamma)-(x-\gamma) \frac{f^{\prime}(\gamma)+f^{\prime}(x)}{2},
\end{gathered}
$$

where $\gamma$ is the initial approximation for a zero of (1.1).

We can rewrite (2.1) in the following form:

$$
\begin{aligned}
x & =\gamma-2 \frac{f(\gamma)}{f^{\prime}(\gamma)}-(x-\gamma) \frac{f^{\prime}(x)}{f^{\prime}(\gamma)}-2 \frac{g(x)}{f^{\prime}(\gamma)} \\
& =c+N(x),
\end{aligned}
$$

where

$$
c=\gamma-2 \frac{f(\gamma)}{f^{\prime}(\gamma)}
$$

and

$$
N(x)=-(x-\gamma) \frac{f^{\prime}(x)}{f^{\prime}(\gamma)}-2 \frac{g(x)}{f^{\prime}(\gamma)} .
$$

Here $N(x)$ is a nonlinear operator.

As in [16], the solution of (2.3) has the series form,

$$
x=\sum_{i=0}^{\infty} x_{i}
$$

The nonlinear operator $N(x)$ can be decomposed as it has been shown in [16]. Also the series $\sum_{i=0}^{\infty} x_{i}$ converges absolutely and uniformly to a unique solution of equation (2.3) if the nonlinear operator

$$
N(x)=N\left(\sum_{i=0}^{\infty} x_{i}\right)=N\left(x_{0}\right)+\sum_{i=1}^{\infty}\left\{N\left(\sum_{j=0}^{i} x_{j}\right)\right\}
$$


is a contraction.

Combining (2.3), (2.6) and (2.7), we have

$$
\sum_{i=0}^{\infty} x_{i}=c+N\left(x_{0}\right)+\sum_{i=1}^{\infty}\left\{N\left(\sum_{j=0}^{i} x_{j}\right)\right\} .
$$

Thus we have the following iterative scheme:

$$
\begin{aligned}
x_{0}= & c \\
x_{1}= & N\left(x_{0}\right), \\
x_{2}= & N\left(x_{0}+x_{1}\right), \\
& \vdots \\
x_{n+1}= & N\left(x_{0}+x_{1}+\ldots+x_{n}\right), \quad n=1,2, \ldots .
\end{aligned}
$$

Then

$$
\begin{aligned}
& x_{1}+x_{2}+\cdots+x_{n+1} \\
& =N\left(x_{0}\right)+N\left(x_{0}+x_{1}\right)+\cdots+N\left(x_{0}+x_{1}+\cdots+x_{n}\right), \quad n=1,2, \ldots,
\end{aligned}
$$

and

$$
x=c+\sum_{i=1}^{\infty} x_{i} .
$$

From (2.4), (2.5) and (2.9), we have

$$
x_{0}=c=\gamma-2 \frac{f(\gamma)}{f^{\prime}(\gamma)},
$$

and

$$
\begin{aligned}
x_{1} & =N\left(x_{0}\right) \\
& =-\left(x_{0}-\gamma\right) \frac{f^{\prime}\left(x_{0}\right)}{f^{\prime}(\gamma)}-2 \frac{g\left(x_{0}\right)}{f^{\prime}(\gamma)} \\
& =-2 \frac{f\left(x_{0}\right)}{f^{\prime}(\gamma)} .
\end{aligned}
$$

It follows from (2.4), (2.9) and (2.10), that

$$
\begin{aligned}
x & \approx x_{0} \\
& =c \\
& =\gamma-2 \frac{f(\gamma)}{f^{\prime}(\gamma)} .
\end{aligned}
$$

This enables us to suggest the following method for solving the nonlinear equation (1.1). 
Algorithm 2.1. For the given $x_{0}$ compute the approximate solution $x_{n+1}$ by the iterative schemes:

$$
x_{n+1}=x_{n}-2 \frac{f\left(x_{n}\right)}{f^{\prime}\left(x_{n}\right)}, \quad f^{\prime}\left(x_{n}\right) \neq 0, \quad n=0,1,2, \ldots,
$$

which is known as the generalized Newton's formula and is quadratically convergent.

Again by using (2.4), (2.5), (2.9) and (2.10), we conclude that

$$
\begin{aligned}
x & \approx x_{0}+x_{1} \\
& =c+x_{1} \\
& =x_{0}+N\left(x_{0}\right) \\
& =\gamma-2 \frac{f(\gamma)}{f^{\prime}(\gamma)}-2 \frac{f\left(x_{0}\right)}{f^{\prime}(\gamma)}
\end{aligned}
$$

Using (2.14), we can suggest the following two-step iterative method for solving nonlinear equation (1.1) as:

Algorithm 2.2. For the given $x_{0}$ compute the approximate solution $x_{n+1}$ by the iterative schemes:

$$
\begin{aligned}
y_{n} & =x_{n}-2 \frac{f\left(x_{n}\right)}{f^{\prime}\left(x_{n}\right)}, \quad f^{\prime}\left(x_{n}\right) \neq 0, \quad n=0,1,2, \ldots, \\
x_{n+1} & =y_{n}-2 \frac{f\left(y_{n}\right)}{f^{\prime}\left(x_{n}\right)} .
\end{aligned}
$$

Again, using (2.5), (2.11) and (2.12), we can calculate

$$
\begin{aligned}
N\left(x_{0}+x_{1}\right) & =-\left(x_{0}+x_{1}-\gamma\right) \frac{f^{\prime}\left(x_{0}+x_{1}\right)}{f^{\prime}(\gamma)}-2 \frac{g\left(x_{0}+x_{1}\right)}{f^{\prime}(\gamma)} \\
& =-2 \frac{f\left(x_{0}\right)}{f^{\prime}(\gamma)}-2 \frac{f\left(x_{0}+x_{1}\right)}{f^{\prime}(\gamma)} .
\end{aligned}
$$

From (2.10), (2.11), (2.12) and (2.15), we get

$$
\begin{aligned}
x & \approx x_{0}+x_{1}+x_{2} \\
& =c+N\left(x_{0}\right)+N\left(x_{0}+x_{1}\right) \\
& =\gamma-2 \frac{f(\gamma)}{f^{\prime}(\gamma)}-4 \frac{f\left(x_{0}\right)}{f^{\prime}(\gamma)}-2 \frac{f\left(x_{0}+x_{1}\right)}{f^{\prime}(\gamma)} .
\end{aligned}
$$

Using (2.16), we can suggest and analyze the following three-step iterative method for solving nonlinear equation (1.1). 
Algorithm 2.3. For a given $x_{0}$, compute the approximate solution by the iterative schemes:

$$
\begin{aligned}
y_{n} & =x_{n}-2 \frac{f\left(x_{n}\right)}{f^{\prime}\left(x_{n}\right)}, \quad f^{\prime}\left(x_{n}\right) \neq 0, \quad n=0,1,2, \ldots, \\
z_{n} & =-2 \frac{f\left(y_{n}\right)}{f^{\prime}\left(x_{n}\right)}, \\
x_{n+1} & =y_{n}-4 \frac{f\left(y_{n}\right)}{f^{\prime}\left(x_{n}\right)}-2 \frac{f\left(y_{n}+z_{n}\right)}{f^{\prime}\left(x_{n}\right)} .
\end{aligned}
$$

\section{Convergence Analysis}

The convergence analysis of Algorithms 2.2 and 2.3 is given in this section.

Theorem 3.1. Assume that the function $f: D \subset \mathbb{R} \rightarrow \mathbb{R}$ for an open interval $D$ has a multiple root $\alpha \in D$ of multiplicity 2. Let $f(x)$ be sufficiently smooth in the neighborhood of the root $\alpha$. Then the order of convergence of the methods defined by Algorithms 2.2 and 2.3 is 2 .

Proof. Let $\alpha$ be a root of $f(x)$ of multiplicity 2 , then by expanding $f\left(x_{n}\right)$ and $f^{\prime}\left(x_{n}\right)$, in Taylor's series about $\alpha$, we obtain

$$
\begin{gathered}
f\left(x_{n}\right)=c_{2} e_{n}^{2}+c_{3} e_{n}^{3}+c_{4} e_{n}^{4}+O\left(e_{n}^{5}\right), \\
f^{\prime}\left(x_{n}\right)=2 c_{2} e_{n}+3 c_{3} e_{n}^{2}+4 c_{4} e_{4}^{3}+5 c_{5} e_{n}^{4}+O\left(e_{n}^{5}\right),
\end{gathered}
$$

where $e_{n}=x_{n}-\alpha$ and $c_{k}=\frac{f^{(k)}(\alpha)}{k !}, k=2,3, \ldots$

Using (3.1) and (3.2), we have

$$
\begin{aligned}
\frac{f\left(x_{n}\right)}{f^{\prime}\left(x_{n}\right)}= & \frac{1}{2} e_{n}-\frac{1}{4} \frac{c_{3}}{c_{2}} e_{n}^{2}+\left(-\frac{1}{2} \frac{c_{4}}{c_{2}}+\frac{3}{8} \frac{c_{3}^{2}}{c_{2}^{2}}\right) e_{n}^{3} \\
& +\left(-\frac{3}{4} \frac{c_{5}}{c_{2}}+\frac{5}{4} \frac{c_{3} c_{4}}{c_{2}^{2}}-\frac{9}{16} \frac{c_{3}^{3}}{c_{2}^{3}}\right) e_{n}^{4}+O\left(e_{n}^{5}\right) .
\end{aligned}
$$

Thus

$$
\begin{aligned}
y_{n}= & x_{n}-2 \frac{f\left(x_{n}\right)}{f^{\prime}\left(x_{n}\right)} \\
= & \alpha+\frac{1}{2} \frac{c_{3}}{c_{2}} e_{n}^{2}+\left(\frac{c_{4}}{c_{2}}-\frac{3}{4} \frac{c_{3}^{2}}{c_{2}^{2}}\right) e_{n}^{3} \\
& +\left(\frac{3}{2} \frac{c_{5}}{c_{2}}-\frac{5}{2} \frac{c_{3} c_{4}}{c_{2}^{2}}+\frac{9}{8} \frac{c_{3}^{3}}{c_{2}^{3}}\right) e_{n}^{4}+O\left(e_{n}^{5}\right) .
\end{aligned}
$$


Expanding $f\left(y_{n}\right)$ by Taylor's series about $\alpha$, we get

$$
\begin{aligned}
f\left(y_{n}\right)= & \frac{1}{4} \frac{c_{3}^{2}}{c_{2}} e_{n}^{4}+\left(\frac{c_{3} c_{4}}{c_{2}}-\frac{3}{4} \frac{c_{3}^{3}}{c_{2}^{2}}\right) e_{n}^{5} \\
& +\left(\frac{3}{2} \frac{c_{3} c_{5}}{c_{2}}-\frac{4 c_{4} c_{3}^{2}}{c_{2}^{2}}+\frac{29}{16} \frac{c_{3}^{4}}{c_{2}^{3}}+\frac{c_{4}^{2}}{c_{2}}\right) e_{n}^{6}+O\left(e_{n}^{7}\right) .
\end{aligned}
$$

Using (3.2), (3.3) and (3.5), the error tem becomes

$$
\begin{aligned}
e_{n+1}= & \frac{1}{2} \frac{c_{3}}{c_{2}} e_{n}^{2}+\left(\frac{c_{4}}{c_{2}}-\frac{7}{8} \frac{c_{3}^{2}}{c_{2}^{2}}\right) e_{n}^{3} \\
& +\left(\frac{3}{2} \frac{c_{5}}{c_{2}}-\frac{3 c_{3} c_{4}}{c_{2}^{2}}+\frac{27}{16} \frac{c_{3}^{3}}{c_{2}^{3}}\right) e_{n}^{4}+O\left(e_{n}^{5}\right) .
\end{aligned}
$$

Now for Algorithm 2.3, we use (3.2) and (3.5) to compute $z_{n}$ as follows

$$
\begin{aligned}
z_{n} & =-2 \frac{f\left(y_{n}\right)}{f^{\prime}\left(x_{n}\right)} \\
& =-c_{3}^{2} e_{n}^{5}+\left(\frac{3}{2} \frac{c_{3}^{3}}{c_{2}}-4 c_{3} c_{4}\right) e_{n}^{6}+O\left(e_{n}^{7}\right) .
\end{aligned}
$$

Using (3.5) and (3.8), we have

$$
\begin{aligned}
y_{n}+z_{n}= & \alpha+\frac{1}{2} \frac{c_{3}}{c_{2}} e_{n}^{2}+\left(\frac{c_{4}}{c_{2}}-\frac{1}{4} \frac{c_{3}^{2}}{c_{2}^{2}}\right) e_{n}^{3} \\
& +\left(\frac{3}{2} \frac{c_{5}}{c_{2}}-\frac{7}{4} \frac{c_{3}^{3}}{c_{2}^{3}}\right) e_{n}^{4}+O\left(e_{n}^{5}\right) .
\end{aligned}
$$

Expanding $f\left(y_{n}+z_{n}\right)$ by Taylor's series about $\alpha$, we obtain

$$
\begin{aligned}
f\left(y_{n}+z_{n}\right)= & \frac{1}{4} \frac{c_{3}^{2}}{c_{2}} e_{n}^{4}+\left(\frac{c_{3} c_{4}}{c_{2}}-\frac{3}{4} \frac{c_{3}^{3}}{c_{2}^{2}}\right) e_{n}^{5} \\
& +\left(\frac{3}{2} \frac{c_{3} c_{5}}{c_{2}}-\frac{4 c_{4} c_{3}^{2}}{c_{2}^{2}}+\frac{29}{16} \frac{c_{3}^{4}}{c_{2}^{3}}+\frac{c_{4}^{2}}{c_{2}}\right) e_{n}^{6}+O\left(e_{n}^{7}\right) .
\end{aligned}
$$

Now using (3.2), (3.4), (3.7) and (3.9), we have the error tem as

$$
\begin{aligned}
e_{n+1}= & \frac{1}{2} \frac{c_{3}}{c_{2}} e_{n}^{2}+\left(\frac{c_{4}}{c_{2}}-\frac{3}{2} \frac{c_{3}^{2}}{c_{2}^{2}}\right) e_{n}^{3} \\
& +\left(\frac{3}{2} \frac{c_{5}}{c_{2}}-\frac{11}{2} \frac{c_{3} c_{4}}{c_{2}^{2}}+\frac{19}{4} \frac{c_{3}^{3}}{c_{2}^{3}}\right) e_{n}^{4}+O\left(e_{n}^{5}\right) . .
\end{aligned}
$$

The last equation shows that the convergence order of Algorithm 2.3 is 2. This completes the proof. 


\section{Numerical Examples}

In this section, we present some numerical examples to demonstrate the performance of the newly developed iterative method. In the following Tables 1-12, we compare our proposed methods (Algorithms 2.2 and 2.3) (NIM1) and (NIM2) with classical Newton's method (NM), generalized Newton's method (GNM), Chun et al. [6, Equations (35) and (36] (BNM1) and (BNM2). All the computations for above mentioned methods are performed using software Maple and $\varepsilon=10^{-10}$ as tolerance and also the following criteria is used for estimating the zero:

(i) $\delta=\left|x_{n+1}-x_{n}\right|<\varepsilon$,

(ii) $\left|f\left(x_{n}\right)\right|<\varepsilon$,

(iii) Maximum numbers of iterations $=500$.

Here $N$ denotes the number of iterations.

Table 1. Comparison for $f_{1}(x)=(x-2)^{2}$

\begin{tabular}{|c|c|l|c|c|c|c|}
\hline$f(x)$ & $x_{\circ}$ & Method & $N$ & $x[k]$ & $f\left(x_{n}\right)$ & $\delta$ \\
\hline & & NM & 10 & 1.9999707031250000 & $8.58 e-10$ & $2.93 e-05$ \\
& & GNM & 1 & 2.0000000000000000 & $0.00 e+00$ & $3.00 e-02$ \\
$f_{1}(x)$ & \multirow{2}{*}{1.97} & BNM1 & 1 & 2.0000000000000000 & $0.00 e+00$ & $3.00 e-02$ \\
& & BNM2 & 1 & 2.0000000000000000 & $0.00 e+00$ & $3.00 e-02$ \\
& & NIM1 & 1 & 2.0000000000000000 & $0.00 e+00$ & $3.00 e-02$ \\
& & NIM2 & 1 & 2.0000000000000000 & $0.00 e+00$ & $3.00 e-02$ \\
\hline
\end{tabular}

Table 2. Comparison for $f_{2}(x)=\arctan ^{2} x$

\begin{tabular}{|c|c|l|c|l|c|c|}
\hline$f(x)$ & $x_{\circ}$ & Method & $\mathrm{N}$ & \multicolumn{1}{c|}{$x[k]$} & $f\left(x_{n}\right)$ & $\delta$ \\
\hline & & NM & 10 & 0.0000195243074874 & $3.81 e-10$ & $1.95 e-05$ \\
& & GNM & 1 & -0.0000053329067398 & $2.84 e-11$ & $2.00 e-02$ \\
$f_{2}(x)$ & \multirow{2}{*}{0.02} & BNM1 & 1 & 0.0000026713622895 & $7.14 e-12$ & $2.00 e-02$ \\
& & BNM2 & 1 & 0.0000026617546045 & $7.08 e-12$ & $2.00 e-02$ \\
& & NIM1 & 1 & -0.0000053343294930 & $2.85 e-11$ & $2.00 e-02$ \\
& & NIM2 & 1 & -0.0000053371757585 & $2.85 e-11$ & $2.00 e-02$ \\
\hline
\end{tabular}

Table 3. Comparison for $f_{3}(x)=\left(e^{x}-4 x^{2}\right)^{2}$

\begin{tabular}{|c|c|l|c|c|c|c|}
\hline$f(x)$ & $x_{\circ}$ & Method & $\mathrm{N}$ & $x[k]$ & $f\left(x_{n}\right)$ & $\delta$ \\
\hline & & NM & 12 & -0.4077725133649210 & $2.72 e-10$ & $4.20 e-06$ \\
& & GNM & 2 & -0.4077767961647673 & $1.16 e-13$ & $3.05 e-04$ \\
$f_{3}(x)$ & \multirow{3}{*}{0.39} & BNM1 & 2 & -0.4077767094044696 & $1.78 e-27$ & $1.69 e-05$ \\
& & BNM2 & 1 & -0.4077777243328640 & $1.59 e-11$ & $1.78 e-02$ \\
& & NIM1 & 2 & -0.4077767992980449 & $1.25 e-13$ & $3.10 e-04$ \\
& & NIM2 & 2 & -0.4077768058455424 & $1.43 e-13$ & $3.22 e-04$ \\
\hline
\end{tabular}


Table 4. Comparison for $f_{4}(x)=x^{3}-x^{2}-x+1$

\begin{tabular}{|c|c|l|c|c|c|c|}
\hline$f(x)$ & $x_{\circ}$ & Method & $\mathrm{N}$ & $x[k]$ & $f\left(x_{n}\right)$ & $\delta$ \\
\hline & & NM & 11 & 1.0000148669755619 & $4.42 e-10$ & $1.49 e-05$ \\
& & GNM & 2 & 1.0000000121033821 & $2.93 e-16$ & $2.20 e-04$ \\
$f_{4}(x)$ & \multirow{2}{*}{1.03} & BNM1 & 1 & 1.0000055621052384 & $6.19 e-11$ & $3.00 e-02$ \\
& & BNM2 & 1 & 1.0000009278935850 & $1.72 e-12$ & $3.00 e-02$ \\
& & NIM1 & 2 & 1.0000000119297000 & $2.85 e-16$ & $2.18 e-04$ \\
& & NIM2 & 2 & 1.0000000115885852 & $2.69 e-16$ & $2.15 e-04$ \\
\hline
\end{tabular}

Table 5. Comparison for $f_{5}(x)=(\sin x-\cos x)^{2}$

\begin{tabular}{|c|c|l|c|c|c|c|}
\hline$f(x)$ & $x_{\circ}$ & Method & $\mathrm{N}$ & $x[k]$ & $f\left(x_{n}\right)$ & $\delta$ \\
\hline & & NM & 12 & 0.7853773818407633 & $8.64 e-10$ & $2.08 e-05$ \\
& & GNM & 2 & 0.7853981633944398 & $1.81 e-23$ & $2.08 e-04$ \\
$f_{5}(x)$ & \multirow{2}{*}{0.7} & BNM1 & 2 & 0.7853981633972462 & $8.17 e-26$ & $1.07 e-04$ \\
& & BNM2 & 2 & 0.7853981633972653 & $6.70 e-26$ & $1.03 e-04$ \\
& & NIM1 & 2 & 0.7853981633944176 & $1.84 e-23$ & $2.09 e-04$ \\
& & NIM2 & 2 & 0.7853981633943728 & $1.89 e-23$ & $2.10 e-04$ \\
\hline
\end{tabular}

Table 6. Comparison for $f_{6}(x)=\left(x^{2}+\ln x^{2}+\sin x^{2}\right)^{2}$

\begin{tabular}{|c|c|l|c|c|c|c|}
\hline$f(x)$ & $x_{\circ}$ & Method & $\mathrm{N}$ & $x[k]$ & $f\left(x_{n}\right)$ & $\delta$ \\
\hline & & NM & 13 & 0.6552172246879896 & $5.83 e-10$ & $4.35 e-06$ \\
& & GNM & 2 & 0.6552215653222547 & $9.30 e-16$ & $1.98 e-04$ \\
$f_{6}(x)$ & \multirow{3}{*}{62} & BNM1 & 2 & 0.6552215708114534 & $2.46 e-30$ & $1.11 e-05$ \\
& & BNM2 & 1 & 0.6552215708114528 & $2.35 e-29$ & $1.48 e-05$ \\
& & NIM1 & 2 & 0.6552215653829742 & $9.09 e-16$ & $1.97 e-04$ \\
& & NIM2 & 2 & 0.6552215655027309 & $8.69 e-16$ & $1.95 e-04$ \\
\hline
\end{tabular}

Table 7. Comparison for $f_{7}(x)=x^{3}-3 x+2$

\begin{tabular}{|c|c|l|c|c|c|c|}
\hline$f(x)$ & $x_{\circ}$ & Method & N & $x[k]$ & $f\left(x_{n}\right)$ & $\delta$ \\
\hline & & NM & 12 & 1.0000149394272540 & $6.70 e-10$ & $1.49 e-05$ \\
& & GNM & 2 & 1.0000000565392868 & $9.59 e-15$ & $5.82 e-04$ \\
$f_{7}(x)$ & \multirow{3}{*}{1.06} & BNM1 & 2 & 1.0000000000000007 & $1.51 e-30$ & $1.94 e-05$ \\
& & BNM2 & 1 & 1.0000033833477819 & $3.43 e-11$ & $6.00 e-02$ \\
& & NIM1 & 2 & 1.0000000554730511 & $9.23 e-15$ & $5.77 e-04$ \\
& & NIM2 & 2 & 1.0000000533910456 & $8.55 e-15$ & $5.66 e-04$ \\
\hline
\end{tabular}

Table 8. Comparison for $f_{8}(x)=\left(x-e^{\cos x}\right)^{2}$

\begin{tabular}{|c|c|l|c|c|c|c|}
\hline$f(x)$ & $x_{\circ}$ & Method & $\mathrm{N}$ & $x[k]$ & $f\left(x_{n}\right)$ & $\delta$ \\
\hline & & NM & 13 & 1.3029548560201398 & $4.26 e-10$ & $9.15 e-06$ \\
& & GNM & 2 & 1.3029638361281054 & $1.39 e-13$ & $9.27 e-04$ \\
$f_{8}(x)$ & \multirow{3}{*}{1.23} & BNM1 & 2 & 1.3029640012159444 & $2.37 e-26$ & $7.09 e-05$ \\
& & BNM2 & 2 & 1.3029640012160073 & $1.40 e-28$ & $4.04 e-05$ \\
& & NIM1 & 2 & 1.3029638401650150 & $1.32 e-13$ & $9.16 e-04$ \\
& & NIM2 & 2 & 1.3029638479906811 & $1.20 e-13$ & $8.94 e-04$ \\
\hline
\end{tabular}


Table 9. Comparison for $f_{9}(x)=(x-\tan x)^{2}$

\begin{tabular}{|c|c|l|l|c|c|c|}
\hline$f(x)$ & $x_{\circ}$ & Method & $\mathrm{N}$ & $x[k]$ & $f\left(x_{n}\right)$ & $\delta$ \\
\hline & & NM & 1 & 0.0416722242070108 & $5.83 e-10$ & $8.33 e-03$ \\
& & GNM & 1 & 0.0333444484140216 & $1.53 e-10$ & $1.67 e-02$ \\
$f_{9}(x)$ & \multirow{2}{*}{0.05} & BNM1 & 1 & 0.0288991908845572 & $6.47 e-11$ & $2.11 e-02$ \\
& & BNM2 & 1 & 0.0255627464167388 & $3.10 e-11$ & $2.44 e-02$ \\
& & NIM1 & 1 & 0.0318809320892903 & $1.17 e-10$ & $1.81 e-02$ \\
& & NIM2 & 1 & 0.0292994996253647 & $7.03 e-11$ & $2.07 e-02$ \\
\hline
\end{tabular}

Table 10. Comparison for $f_{10}(x)=(x-\sin x)^{2}$

\begin{tabular}{|c|c|l|c|c|c|c|}
\hline$f(x)$ & $x_{\circ}$ & Method & $\mathrm{N}$ & $x[k]$ & $f\left(x_{n}\right)$ & $\delta$ \\
\hline & & NM & 1 & 0.0499987998456958 & $4.34 e-10$ & $1.00 e-02$ \\
& & GNM & 1 & 0.0399975996913915 & $1.14 e-10$ & $2.00 e-02$ \\
$f_{10}(x)$ & \multirow{2}{*}{0.06} & BNM1 & 1 & 0.0346644425641644 & $4.82 e-11$ & $2.53 e-02$ \\
& & BNM2 & 1 & 0.0306651148409437 & $2.31 e-11$ & $2.93 e-02$ \\
& & NIM1 & 1 & 0.0382418400691131 & $8.69 e-11$ & $2.18 e-02$ \\
& & NIM2 & 1 & 0.0351448601057440 & $5.23 e-11$ & $2.49 e-02$ \\
\hline
\end{tabular}

Table 11. Comparison for $f_{11}(x)=\left(x^{2}+\sin \frac{x}{5}-\frac{1}{4}\right)^{2}$

\begin{tabular}{|c|c|l|c|c|c|c|}
\hline$f(x)$ & $x_{\circ}$ & Method & $\mathrm{N}$ & $x[k]$ & $f\left(x_{n}\right)$ & $\delta$ \\
\hline & & NM & 11 & 0.4099740214194201 & $3.36 e-10$ & $1.80 e-05$ \\
& & GNM & 2 & 0.4099948386255551 & $8.27 e-12$ & $1.70 e-03$ \\
$f_{11}(x)$ & \multirow{3}{*}{0.37} & BNM1 & 2 & 0.4099920179505115 & $1.55 e-21$ & $2.37 e-04$ \\
& & BNM2 & 2 & 0.4099920179891372 & $2.43 e-33$ & $3.20 e-05$ \\
& & NIM1 & 2 & 0.4099951109809942 & $9.94 e-12$ & $1.78 e-03$ \\
& & NIM2 & 2 & 0.4099957203043393 & $1.42 e-11$ & $1.95 e-03$ \\
\hline
\end{tabular}

Table 12. Comparison for $f_{12}(x)=\left(x^{2}+7 x-30\right)^{2}$

\begin{tabular}{|c|c|l|c|c|c|c|}
\hline$f(x)$ & $x_{\circ}$ & Method & $\mathrm{N}$ & $x[k]$ & $f\left(x_{n}\right)$ & $\delta$ \\
\hline & & NM & 14 & 3.0000018395052141 & $5.72 e-10$ & $1.84 e-06$ \\
& & GNM & 2 & 3.0000000003653009 & $2.26 e-17$ & $6.89 e-05$ \\
$f_{12}(x)$ & \multirow{3}{*}{3.03} & BNM1 & 1 & 3.0000004727268600 & $3.78 e-11$ & $3.00 e-02$ \\
& & BNM2 & 1 & 3.0000000032477361 & $1.78 e-15$ & $3.00 e-02$ \\
& & NIM1 & 2 & 3.0000000003636341 & $2.23 e-17$ & $6.88 e-05$ \\
& & NIM2 & 2 & 3.0000000003603197 & $2.19 e-17$ & $6.84 e-05$ \\
\hline
\end{tabular}

\section{Conclusions}

In the present work, we have proposed two new iterative methods (NIM1) and (NIM2) with convergence order 2 for finding the multiple roots of nonlinear equations. The numerical results presented in the Tables 1-12 given in the previous section reveal that our iterative methods (NIM1) and (NIM2) are even comparable with the methods developed by Chun et al. [6] (BNM1) and 
(BNM2) with convergence order 3. The idea and technique employed in this paper can be developed to higher-order multi-step iterative methods for solving nonlinear equations having multiple roots.

\section{References}

[1] S. Abbasbandy, Improving Newton-Raphson method for nonlinear equations modified Adomian decomposition method, Appl. Math. Comput., 145 (2003), 887-893. doi: 10.1016/S0096-3003(03)00282-0

[2] G. Adomian, Nonlinear Stochastic Systems and Applications to Physics, Kluwer Academic Publishers, Dordrecht, 1989.

[3] E. Babolian, J. Biazar, On the order of convergence of Adomian method, Appl. Math. Comput., 130 (2002), 383-387. doi: 10.1016/S0096-3003(01)00103-5

[4] E. Babolian, J. Biazar, Solution of nonlinear equations by modified Adomian decomposition method, Appl. Math. Comput., 132 (2002), 167-172. doi: 10.1016/S00963003(01)00184-9

[5] C. Chun, Iterative methods improving Newton's method by the decomposition method, Comput. Math. Appl., 50 (2005), 1559-1568. doi: 10.1016/j.camwa.2005.08.022

[6] C. Chun, H.J. Bae, B. Neta, New families of nonlinear third-order solvers for finding multiple roots, Comput. Math. Appl., 57 (2009), 1574-1582. doi: 10.1016/j.camwa.2008.10.070

[7] C. Chun, Y. Ham, A one-parameter fourth-order family of iterative methods for nonlinear equations, Appl. Math. Comput., 189 (2007), 610-614. doi: 10.1016/j.amc.2006.11.113

[8] C. Chun, B. Neta, A third-order modification of Newton's method for multiple roots, Appl. Math. Comput., 211 (2009), 474-479. doi: 10.1016/j.amc.2009.01.087

[9] C. Chun, B. Neta, Basin of attraction for Zhou-Chen-Song fourth order family of methods for multiple roots, Math. Comput. Simulation, 109 (2015), 74-91. doi: 10.1016/j.matcom.2014.08.005

[10] V. Daftardar-Gejji, H. Jafari, An iterative method for solving nonlinear functional equations, J. Math. Anal. Appl., 316 (2006), 753-763. doi: 10.1016/j.jmaa.2005.05.009

[11] V.I. Hasanov, I.G. Ivanov, G. Nedzhibov, A new modification of Newton's method, Appl. Math. Engin. Econom. (Sozopol, 2001), pp. 278-286, Heron Press, Sofia, 2002

[12] H.H.H. Homeier, On Newton-type methods for multiple roots with cubic convergence, $J$. Comput. Appl. Math., 231 (2009), 249-254. doi: 10.1016/j.cam.2009.02.006

[13] E. Isaacson, H.B. Keller, Analysis of Numerical Methods, John Wiley \& Sons, Inc., New York, 1966.

[14] B. Neta, C. Chun, On a family of Laguerre methods to find multiple roots of nonlinear equations, Appl. Math. Comput., 219 (2013), 10987-11004. doi: 10.1016/j.amc.2013.05.002

[15] B. Neta, C. Chun, M. Scott, On a development of iterative methods for multiple roots, Appl. Math. Comput., 224 (2013), 358-361. doi: 10.1016/j.amc.2013.08.077 
[16] M.A. Noor, K.H. Noor, Some iterative schemes for nonlinear equations, Appl. Math. Comput., 183 (2006), 774-779. doi: 10.1016/j.amc.2006.05.084

[17] N. Osada, An optimal multiple root-finding method of order three, J. Comput. Appl. Math., 51 (1994), 131-133. doi: 10.1016/0377-0427(94)00044-1

[18] E. Schröder, Über unendlich viele algorithmen zur auflösung der gleichungen, Math. Ann., 2 (1870), 317-365. doi: 10.1007/BF01444024. 